\title{
Emotional decoding
}

Sharon Cox

1 London South Bank University

A mental ability of a person to process and accurately recognise auditory, linguistic, visual, and physical cues that convey emotion. 